\title{
metilene: fast and sensitive calling of differentially methylated regions from bisulfite sequencing data
}

\author{
Frank Jühling, ${ }^{1,2,7}$ Helene Kretzmer, ${ }^{1,2,7}$ Stephan H. Bernhart, ${ }^{1,2}$ Christian Otto, ${ }^{1,2}$ \\ Peter F. Stadler, $2,3,4,5,6$ and Steve Hoffmann ${ }^{1,2}$ \\ ${ }^{1}$ Transcriptome Bioinformatics Group, LIFE - Leipzig Research Center for Civilization Diseases, University of Leipzig, 04107 Leipzig, \\ Germany; ${ }^{2}$ Interdisciplinary Center for Bioinformatics and Bioinformatics Group, Faculty of Computer Science, University of Leipzig, \\ 04107 Leipzig, Germany; ${ }^{3}$ RNomics Group, Fraunhofer Institute for Cell Therapy and Immunology - IZI, 04103 Leipzig, Germany; \\ ${ }^{4}$ Santa Fe Institute, Santa Fe, New Mexico 87501, USA; ${ }^{5}$ Department of Theoretical Chemistry, University of Vienna, 1090 Vienna, \\ Austria; ${ }^{6}$ Max Planck Institute for Mathematics in Sciences, 04103 Leipzig, Germany
}

\begin{abstract}
The detection of differentially methylated regions (DMRs) is a necessary prerequisite for characterizing different epigenetic states. We present a novel program, metilene, to identify DMRs within whole-genome and targeted data with unrivaled specificity and sensitivity. A binary segmentation algorithm combined with a two-dimensional statistical test allows the detection of DMRs in large methylation experiments with multiple groups of samples in minutes rather than days using off-the-shelf hardware. metilene outperforms other state-of-the-art tools for low coverage data and can estimate missing data. Hence, metilene is a versatile tool to study the effect of epigenetic modifications in differentiation/development, tumorigenesis, and systems biology on a global, genome-wide level. Whether in the framework of international consortia with dozens of samples per group, or even without biological replicates, it produces highly significant and reliable results.
\end{abstract}

[Supplemental material is available for this article.]

As one of the most important mechanisms of epigenetic control, localized differential methylation has been associated with a wide variety of phenotypes and conditions. These include cell differentiation (Cortese et al. 2011; Sheaffer et al. 2014), tissue type and age (Day et al. 2013), and pain sensitivity (Bell et al. 2014). Differences in DNA methylation levels have also been connected to many different diseases like diabetes (Nilsson et al. 2014) or Alzheimer's disease (De Jager et al. 2014). Furthermore, differential DNA methylation plays a role in many cancers, such as medulloblastoma (Hovestadt et al. 2014) and B-cell lymphoma (Kretzmer et al. 2015), and connects risk factors like age (Teschendorff et al. 2010) and smoking (Teschendorff et al. 2015) with cancer.

Whole-genome bisulfite sequencing experiments (WGBS) and targeted protocols, e.g., reduced representation bisulfite sequencing (RRBS), made it possible to study cytosine methylation landscapes at single CpG resolution. Decreasing costs and a high availability of next-generation sequencing (NGS) facilities have made it feasible for a rapidly growing research community to study this important epigenetic layer. The identification of differentially methylated regions (DMRs) between different conditions in large groups of samples requires accurate and efficient algorithms. At present, this is a serious bottleneck in methylome analysis.

The objective problem of finding DMRs has two dimensions: The first dimension is to find a genomic region such that, in the second dimension, the individuals of two groups are significantly distinct in their methylation levels. Current solutions typically use pooled data and employ beta-binomial distributions or regression

\footnotetext{
${ }^{7}$ These authors contributed equally to this work. Corresponding author: steve@bioinf.uni-leipzig.de Article published online before print. Article, supplemental material, and publication date are at http://www.genome.org/cgi/doi/10.1101/gr.196394.115.
}

models fitted to single CpG methylation rates. After testing single CpGs for differential methylation (DMC), significant DMCs are merged to DMRs using various approaches (Akalin et al. 2012; Hebestreit et al. 2013; Park et al. 2014; Stockwell et al. 2014; Sun et al. 2014).

\section{Results}

The segmentation algorithm

We present a segmentation algorithm to detect DMRs between single samples as well as in groups of samples (Fig. 1A). As a distinguishing feature, it does not make assumptions on underlying distributions or background models and is applicable to WGBS as well as RRBS data without further parameter adjustments. In contrast to other approaches, we propose a scoring model to find maximum intergroup methylation differences within a genomic interval of minimum length in combination with a nonparametric test. Our approach, based on a circular binary segmentation (CBS) (Siegmund 1986; Olshen et al. 2004), scans for pairs of change points within the mean difference signal (MDS), i.e., difference of $\mathrm{CpG}$-wise mean methylation level in the groups, delimiting a region with homogeneous methylation difference. Subsequently, intervals are tested for similarity using a twodimensional Kolmogorov-Smirnov test (2D-KS test) (Fasano and Franceschini 1987). Initially, the genome is presegmented to avoid calling DMRs containing long stretches without methylation

(C) 2016 Jühling et al. This article is distributed exclusively by Cold Spring Harbor Laboratory Press for the first six months after the full-issue publication date (see http://genome.cshlp.org/site/misc/terms.xhtml). After six months, it is available under a Creative Commons License (Attribution-NonCommercial 4.0 International), as described at http://creativecommons.org/licenses/by$\mathrm{nc} / 4.0 /$. 
A
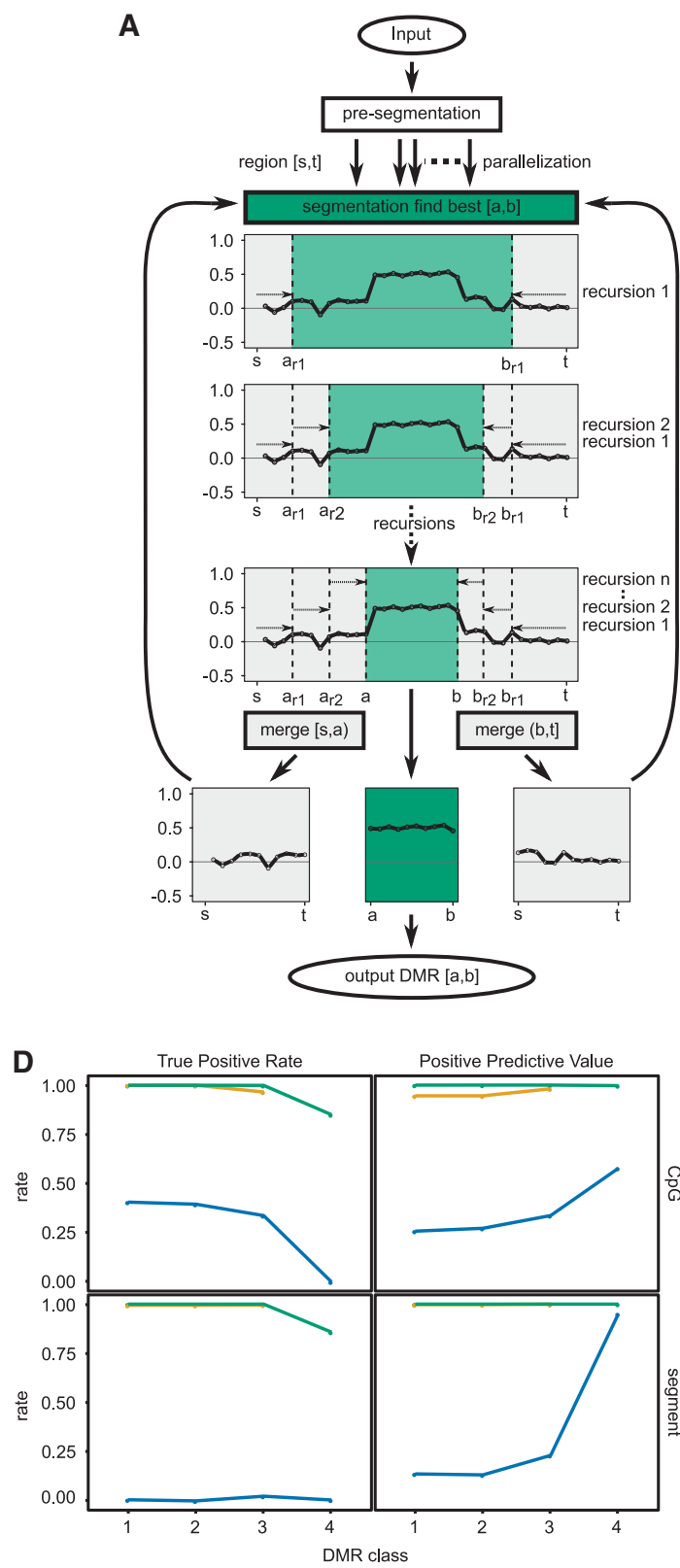

B

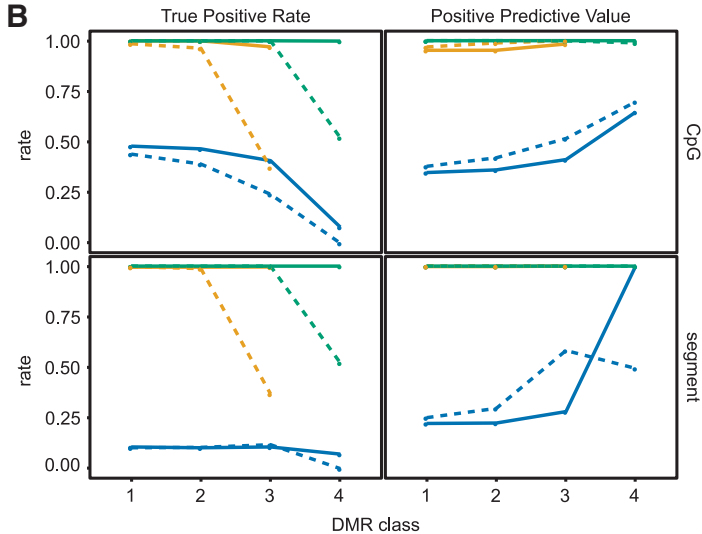

C background 1 \& DMR class 1 background 2 \& DMR class 2

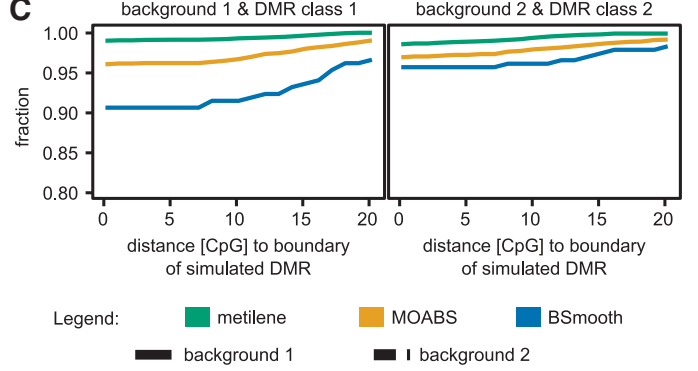

E

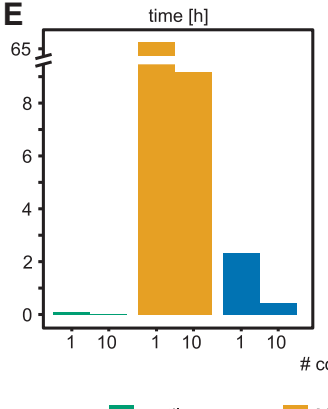

metilene

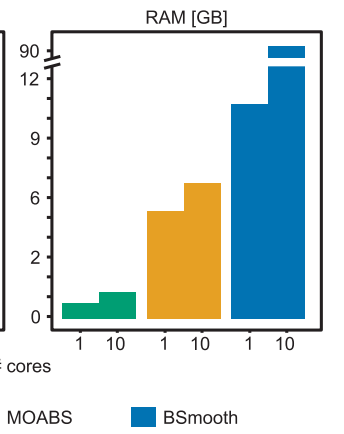

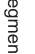

西 
BiSeq (Hebestreit et al. 2013) (v1.2.5), using artificial and real-life data.

\section{Performance tests on artificial data}

We simulated DMRs for the human Chromosome 10 with different backgrounds and degrees of methylation difference, resulting in eight data sets with different levels of complexity (Supplemental Fig. S1; Methods). Two groups with 10 samples each were simulated. A performance evaluation in terms of the true positive rate (TPR) and positive predictive value (PPV) was carried out for CpGs within simulated and predicted DMRs as well as for predicted DMRs overlapping with $\geq 50 \%$ of simulated DMRs.

Almost all DMRs with larger methylation differences (DMR classes 1 and 2) were correctly predicted by metilene and MOABS on both the CpG (Supplemental Table S1) and the DMR level (Supplemental Table S2). With a TPR below 0.5, BSmooth had difficulties identifying simulated DMRs (Fig. 1B). For DMRs with smaller methylation differences (DMR class 3) in regions with difficult background methylation (background 2), the advantage of metilene was more apparent: While the TPR of MOABS dropped below $40 \%$, metilene still reported $99.8 \%$ of the DMRs. A similar TPR was achieved by metilene for the most challenging DMR class 4 in background 1 . A drop in sensitivity of metilene (TPR $\approx 0.5$ ) was only observed for the complicated background. MOABS did not predict any class 4 DMRs. metilene showed a PPV $\geq 0.989$ in all scenarios.

metilene predicted the starts and ends of DMRs (Fig. 1C; Supplemental Fig. S2) within a very small margin of error, independent of the background type and the DMR class. MOABS performed less accurately for the simple data. Likely due to the efficient smoothing step, BSmooth improved with more complicated background methylation. To verify the robustness of predictions, we simulated low coverage data. Here, metilene came up top in both PPV and TPR (Fig. 1D). In addition, different levels of noise were introduced (Methods). Except for very high noise, MOABS and metilene performed comparably (Supplemental Fig. S3).

On a single core, metilene used $\sim 4$ min to analyze the simulated data set (Chromosome 10) with $2 \times 10$ samples. The runnerup with respect to the results, MOABS, needed $>65 \mathrm{~h}$ to perform the same task, whereas BSmooth took $2.3 \mathrm{~h}$. The memory consumption of metilene was $<1 \mathrm{~GB}$, whereas MOABS (5.4 GB) and BSmooth (10.7 GB) used substantially more RAM. On 10 cores, metilene used $\sim 1 \mathrm{~min}$, while MOABS took $\sim 9 \mathrm{~h}$. BSmooth used $23 \mathrm{~min}$. The memory consumption of metilene and MOABS increased only slightly, while BSmooth used $>90$ GB in the multithreaded mode (Fig. 1E).

We explored the performance of metilene on RRBS data and found similar results without further parameter adjustments. We added another specialized RRBS tool, i.e., BiSeq, and showed that metilene still performs favorably regarding the prediction power (Supplemental Tables S3, S4; Supplemental Fig. S4), boundary detection (Supplemental Fig. S5), and runtime and memory requirements (Supplemental Tables S5, S6). In some test scenarios, BiSeq and metilene showed comparable results regarding TPRs. However, BiSeq did not reach metilene's PPV. The results of MOABS and BSmooth on RRBS data are comparable to the results on WGBS data.

\section{DMR calling on biological data}

A human WGBS (Hovestadt et al. 2014) data set comprising 12 samples from human medulloblastomas and eight controls was re- trieved to compare the three tools, i.e., BSmooth, MOABS, and metilene, on real data. For the genome-wide analysis of the WGBS data set, our tool used 10 min on 10 cores, and the peak memory usage was $88.3 \mathrm{MB}$. Because of the memory and runtime requirements of MOABS and BSmooth (Supplemental Table S7), the qualitative comparison of the results had to be restricted to Chromosome 10.

Among the tools tested, metilene found the highest number of DMRs $(n=4602)$, followed by MOABS $(n=2108)$ and BSmooth ( $n=1935)$ (Fig. 2A). This observation is in line with the observed advantages in sensitivity in the simulations. The distribution of additional DMRs detected by metilene peaks around mean methylation differences between 0.2 and 0.3 (Fig. 2B). MOABS has difficulties reporting DMRs with smaller differences, and BSmooth reports only a rather small number of DMRs with higher differences. Using an independent nonparametric test (Wilcoxon) confirms that the DMRs exclusively reported by metilene are significantly differential. In general, metilene's unique DMRs tend to have lower $P$-values than MOABS or BSmooth (Fig. 2C). We found that some of metilene's unique DMRs do not only show a high absolute methylation difference but are also long (Fig. 2D). To test whether the DMRs exclusively reported by metilene can be confirmed with respect to the estimated mean methylation difference, and thus the homogeneity inside the predicted boundaries, we compared the WGBS results to matched 450k methylation arrays. The high correlation coefficient $(r=0.96)$ indicates the good reproducibility of metilene predictions (Fig. 2E). An example of a DMR exclusively found by metilene is shown on Figure 2F. It is located in an intragenic region and shows a strong difference level.

Hovestadt et al. (2014) reported 39 promoter downstream correlating regions (pdCRs) on Chromosome 10, i.e., differential methylated regions correlating with gene expression. Of these 39 pdCRs, 29 were retrieved by metilene, whereas MOABS reported only 21 and BSmooth reported 12. Most pdCRs (seven of 10) not reported by metilene contain large stretches of $>300 \mathrm{nt}$ between CpG sites, whereas the remaining three show highly heterogeneous intra-group methylation levels. With the exception of a single BSmooth prediction, all pdCRs reported by BSmooth and MOABS were also detected by metilene.

\section{Discussion}

In summary, metilene does not rely on any assumptions on underlying distributions or background models, nor on joining DMCs. Thus it is able, in contrast to MOABS and BSmooth, to analyze sample pairs without replicates (Supplemental Fig. S6). Furthermore, it can estimate missing methylation data (Supplemental Fig. S7) to include CpG positions not fully covered in all samples. Additionally, it features modes for testing differential methylation in user-defined regions and finding DMCs.

Despite its superior performance in terms of sensitivity, specificity, and accuracy on simulated data, metilene achieved a speed-up of nearly $1000 \times$ on a single core of a cluster machine compared to MOABS, the only remaining competitor after analyzing the DMR prediction results of all tools (Supplemental Table S5). On a single core of a desktop machine, metilene was able to process the human WGBS set with eight versus 12 human samples in $\sim 15 \mathrm{~min}$. Speed and memory requirements are an important issue concerning future studies. We compared all tools and found only metilene to be prepared for larger sample sizes (Supplemental Table S8). Although metilene still performed as well for small group sizes, MOABS and BSmooth took 4.5 and 18.75 d, respectively, to finish the eight versus eight run. Furthermore, although

\section{Genome Research}

www.genome.org 

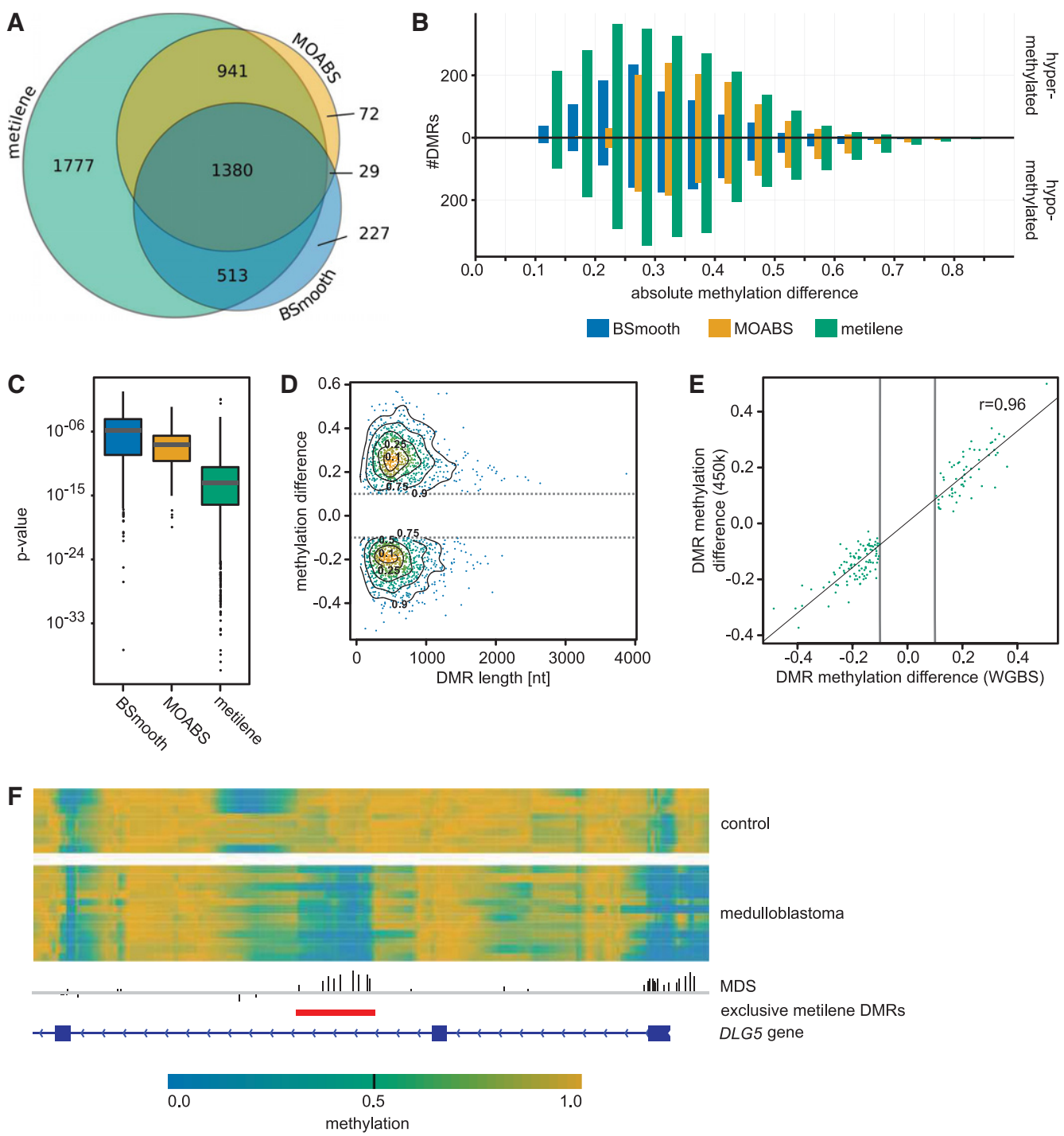

Figure 2. (A) Venn diagram of DMRs found by metilene, BSmooth, and MOABS in the WGBS medulloblastoma data on human Chromosome 10. (B) Count of DMRs exclusively found by metilene, BSmooth, and MOABS binned into methylation difference classes. (C) Box plots of $P$-values of exclusive DMRs using an independent Wilcoxon test. $(D)$ Scatter plot of length and mean methylation differences of DMRs exclusively reported by metilene. Isoclines indicate their distribution, while labels denote the fraction of DMRs found inside the respective area. Note the minimum methylation cutoff at 0.1 (gray line). (E) Correlation of mean difference of exclusive metilene DMRs and 450k methylation beta values. The plot shows all DMRs covered by at least two probes on the array. $(F)$ Figure of the DLG5 gene containing a DMR (red line) exclusively found by metilene. Methylation rates of control (top) and medulloblastoma (below) are heatmap color-coded, indicating low methylation rates in blue and high methylation rates in yellow. The MDS is shown above the DMR annotation and the gene annotation (bottom).

metilene's memory consumption was constantly low $(<150 \mathrm{MB})$ and independent of the number of samples, MOABS and BSmooth consumed between 17 and 300 GB RAM for the same tasks. Both the 16 versus 16 and the 50 versus 50 run could be performed only for metilene due to time/memory issues of both other tools. The results impressively demonstrated the future-proof design of metilene that is capable of dealing with large sample sizes without a large loss of performance. Although we have demonstrated that metilene works well on lowly covered data (sevenfold), we still recommend to provide our tool with methylation rates calculated from 15 reads or more (cf. Ziller et al. 2015).

The nonparametric test at the heart of the strategy integrates two dimensions, i.e., for each given genomic interval (first dimension) the methylation signal of all samples (second dimension).
Our simulations have shown that this method outperforms alternative tools in particular in situations in which group-wise methylation differences are subtle and/or the methylation background is variant. This capability comes in handy when dealing with contaminated or heterogeneous samples. Therefore, metilene seems to be especially suitable for DMR prediction in cancer samples or other samples with a superposition of different methylation signals.

\section{Methods}

\section{Data acquisition}

The biological data that were used within this study are publicly available and were published in the context of the Pediatric Brain 
Cancer project (PBCA-DE) of the International Cancer Genome Consortium (ICGC) through the following submitter IDs: ICGC_MB2, ICGC_MB6, ICGC_MB7, ICGC_MB15, ICGC_MB19, ICGC_ MB24, ICGC_MB26, ICGC_MB32, ICGC_MB38, ICGC_MB40, ICGC_MB49, ICGC_MB51，ICGC_A1，ICGC_A2， ICGC_A3， ICGC_A4， ICGC_F1， ICGC_F2, ICGC_F3, and ICGC_F4. We used ENCODE RRBS data (ENCODE: ENCSR000DFT) and an ENCODE chromatin state segmentation, both of GM12878, for data simulations.

\section{Foundations}

The segmentation is based on a circular binary segmentation (Olshen et al. 2004) in conjunction with a scoring function similar to Siegmund (1986) and Zhang and Siegmund (2012). In a first step, the mean difference signal for each CpG (MDS),

$$
\text { MDS }=\frac{1}{|A|} \sum_{\gamma \in A} p_{\gamma}-\frac{1}{|B|} \sum_{\delta \in B} p_{\delta}
$$

is calculated. Here, $A, B$ are the sample groups to be compared and $p_{\gamma}\left(p_{\delta}\right)$ is the methylation level of the given CpG position in sample $\gamma(\delta)$.

We use a slightly modified scoring function to account for decreasing values in the MDS:

$$
Z_{s, t}=\frac{[|S(a, b)|-(b-a) \cdot|S(s, t)| /(t-s)]^{2}}{(b-a)[1-(b-a) /(t-s)]},
$$

where $Z_{s, t}(a, b)$ denotes the score of window $[a, b]$ in segment $[s, t]$ and $S(i, j)$ the sum of the MDS for the interval $[i, j]$.

$$
S(i, j)=\sum_{0}^{j} \mathrm{MDS}-\sum_{0}^{i} \mathrm{MDS}
$$

The window $\left[a_{\max }, b_{\max }\right]$ with the maximal increase or decrease in the MDS within segment $[s, t]$ is given by

$$
Z_{\max }(a, b)=\max _{s \leq a<b \leq t}\left|Z_{s, t}(a, b)\right| .
$$

Since we consider all samples of both groups, we have slightly modified the originally proposed formula. By maximizing, we find the window in $[s, t]$ that shows the maximal possible change in the MDS. This window is marked as a potential DMR.

The statistical significance of potential DMRs is assessed by a two-dimensional version of the Kolmogorov-Smirnov test (Fasano and Franceschini 1987) to calculate $P$-values during segmentation and to use it as a termination criterion during recursive segmentations. The output of metilene provides the adjusted and unadjusted $P$-value for the 2D-KS test and additionally the results of an independent Mann-Whitney $U$ test.

\footnotetext{
Algorithm

Initially, the genome is presegmented into regions that do not have subintervals longer than $t_{\text {dist }}$ (default: $300 \mathrm{nt}$ ) without methylation information. The presegmented regions are recursively segmented. First, the window $[a, b]$ with the maximal absolute change in the MDS in a region $[s, t]$ is identified via $Z_{\max }$ (Equation 3 ). This results in three subregions: $[s, a-1],[a, b]$, and $[b+1, t]$. The effect of different $t_{\text {dist }}$ settings is shown in Supplemental Table S9. The parameter affects the performance of metilene only slightly as long as it is kept in the range $\leq 500 \mathrm{nt}$. For the sake of biological interpretation, we do not recommend to include longer stretches of the genome without any methylation information.

Second, termination criteria are checked for all three subregions. Every subregion that fulfills none of the termination criteria
}

is further segmented, starting with the first step. We use two distinct termination criteria: (1) number of CpGs $<t_{\min }$ (default: $10)$; and (2) a $P$-value-based termination criterion, i.e., the $P$-value of a subregion must be larger than the $P$-value of the parental region. To save computational time, the $P$-value of a region is not calculated if (1) the segment contains a window of $t_{\text {min }}$ consecutive CpGs with a much weaker MDS than the total segment; or if (2) the signs of MDS have no major trend, i.e., we find a balanced number of both positive and negative values. In such regions, segmentation is directly continued.

Please note that except for the $t_{\min }$ criteria, there are no additional constraints or parameters for the minimum length of an interval $[a, b]$ or $[s, t]$. The effect of different $t_{\min }$ settings is shown in Supplemental Table S10. The parameter does affect the performance of metilene only in WGBS data when it is set to large window sizes.

Third, after the termination of the recursion, the subregion associated with the lowest $P$-value is flagged as a potential DMR. All surrounding subregions are merged and recursively segmented again (see Supplemental Fig. S8 for the pseudocode).

\section{Missing data estimation}

Methylation rates of missing data ( $p_{\text {mis }}$ ) are estimated from a beta distribution $p_{\text {mis }} \sim \operatorname{Beta}(\alpha, \beta)$. The parameters $\alpha$ and $\beta$ are calculated from the mean $\left(\mu_{r}\right)$ and variance $\left(\sigma_{r}^{2}\right)$ of the remaining methylation rates at the corresponding $\mathrm{CpG}$ position:

$$
\begin{gathered}
\alpha=\left(\frac{\mu_{r}\left(1-\mu_{r}\right)}{\sigma_{r}^{2}}-1\right) \mu_{r} \\
\beta=\left(1-\mu_{r}\right)\left(\frac{\mu_{r}\left(1-\mu_{r}\right)}{\sigma_{r}^{2}}-1\right) .
\end{gathered}
$$

Positions with more than a user-defined number of missing values are omitted.

\section{Data simulation}

All DNA methylation simulations were performed on the human Chromosome 10 using a Beta-Binomial approach implemented in R (R Core Team 2013) to simulate both biological methylation and the sequencing step. The simulated data that were used for the benchmarks are available at http://www.bioinf.uni-leipzig. de/Software/metilene/. The scripts and parameters to simulate these data from scratch can also be found in the Supplemental Material.

We simulated two different backgrounds to account for promoter and nonpromoter differences. Since the overall sequence composition in promoters is expected to be different from nonpromoter regions, and to do the simulation in a more realistic fashion, we used the ENCODE chromatin state segmentation of a lymphoblastoid cell line (GM12878) (Ernst and Kellis 2010; Ernst et al. 2011; Raney et al. 2011) to obtain a set of real promoter and nonpromoter regions on Chromosome 10. The GM12878 segmentation has only been used in the simulation and for this specific purpose.

For the 20 simulated samples, methylation rates $p$ were drawn from beta distributions (Beta) with parameters $\alpha$ and $\beta$ to obtain $p \sim \operatorname{Beta}(\alpha, \beta)$. The distributions of the two simulated methylation backgrounds (Supplemental Table S11) are visualized in Supplemental Figure S1A.

Junctions between the nonpromoter and the promoter backgrounds were blurred using a weighted, local polynomial regression fitting (loess; span value of 0.2). Specifically, the methylation rate of 25-50 CpGs (uniform sample) around each junction

\section{Genome Research}

www.genome.org 
was smoothed by setting it to the average of the raw and the fitted value.

Subsequently, an artificial read coverage $n$ was assigned to the CpGs by sampling from a normal distribution $(N)$ with mean $\mu=30$ and standard deviation $\sigma=5$. The minimum required coverage was set to $\varepsilon=15$ reads. For the simulation of lowly methylated data, the parameters were set to $\mu=7, \sigma=2$, and $\varepsilon=3$ :

$$
\begin{aligned}
& n^{*} \sim N\left(\mu, \sigma^{2}\right), \\
& n=\min \left(n^{*}, \varepsilon\right) .
\end{aligned}
$$

To determine the number of these reads with CpGs indicating methylation, $m \sim B(n, p)$ was drawn from a binomial distribution $(B)$ with parameters $n$ and $P$ for coverage and methylation rate, respectively.

To simulate the Foreground, i.e., the DMRs, the 20 samples were split into two groups of 10 samples. A total of 1000 DMRs were introduced into the simulated background of each group. We introduced an equal number of hypermethylated DMRs into promoter (500) and hypomethylated DMRs into nonpromoter regions (500). To generate the DMRs, the parameters $\alpha$ and $\beta$ of the background where swapped, i.e., a DMR within a promoter region was simulated using the beta-distribution for the nonpromoter regions and vice versa. To account for DMRs of different intensities, the values were mixed from both beta distributions and multiplied with different mixing factors $C$,

$$
p \sim \operatorname{Beta}(\alpha, \beta) \cdot c+\operatorname{Beta}(\beta, \alpha) \cdot(1-c),
$$

to generate a total of four different DMR sets. The mixture factors $c$ are given in Supplemental Table S12, and all resulting distributions of DMR classes 1-4 are visualized in Supplemental Figure S1B. Again, the number of reads without conversion was drawn from a binomial distribution.

Reduced representation bisulfite sequencing data were simulated in a similar fashion as the WGBS data. To make our simulation more realistic, we used the regions covered by the publicly available RRBS data set of GM12878. In total, we placed 200 DMRs, 100 DMRs in promoters and 100 DMRs in nonpromoters, with a maximum length of $40 \mathrm{nt}$ inside these regions using the same parameters as for the WGBS simulations.

One of the artificial data sets (background 1 and class 1 DMRs) was used to perform the noise analysis. All DMRs were gradually distorted by replacing 10\%-90\% of all methylation rates with uniformly distributed methylation rates $p_{r} \in[0,1]$ for all samples.

The data set containing 10 versus 10 samples of class 1 DMRs on background 1 was simulated, and methylation values were removed at a probability of $10 \%-90 \%$ to resemble data sets with different degrees of missing values.

\section{Performance evaluation}

All segmentation tools were run in their default settings; and for MOABS, the maximal distance between two consecutive DMCs to be considered in a DMR (maxDistConsDmcs option) was set to $300 \mathrm{nt}$ to be comparable to metilene and BSmooth. Because BSmooth terminated with a runtime error, local corrections were switched off for the RRBS data analysis (local.correct $=F$ ). Predicted DMRs were filtered to a minimal absolute methylation difference (average MDS) larger than 0.1, which is in accordance with the cutoff recommended also by BSmooth. By default, BSmooth reports only significant DMRs. The output of MOABS and metilene was filtered for DMRs with an (adjusted) $P$-value $\leq$ 0.05 . The performance was evaluated in terms of CpG-wise and DMR-wise true positive rates (TPR) and positive predictive values
(PPV). In the following we distinguish between sets of CpGs $(C)$ and sets of CpGs within DMRs $(D)$. A set of DMRs is denoted by $(\mathcal{D})$. The sets of simulated and predicted CpGs inside simulated and predicted DMRs are defined by

$$
\begin{gathered}
C_{\text {sim }}=\left\{C \in \underset{D \in \mathcal{D}_{\text {sim }} D}{\cup} D,\right. \\
C_{\text {pred }}=\left\{C \in \underset{\left.D \in \mathcal{D}_{\text {pred }} D\right\} .}{\cup} .\right.
\end{gathered}
$$

Furthermore, we define true positive (TP) CpGs $\left(C_{\mathrm{TP}}\right)$ as those CpGs in simulated DMRs that were correctly predicted by the segmentation tool. This definition includes all those CpGs that are in the overlap between simulated and predicted DMRs. Thus,

$$
C_{\mathrm{TP}}=C_{\text {sim }} \cap C_{\text {pred }}
$$

is the set of true positive CpGs. We calculate

$$
\begin{aligned}
& \operatorname{PPV}_{\mathrm{CpG}}=\left|C_{\mathrm{TP}}\right| /\left|C_{\text {pred }}\right| \\
& \mathrm{TPR}_{\mathrm{CpG}}=\left|C_{\mathrm{TP}}\right| /\left|C_{\text {sim }}\right|
\end{aligned}
$$

to obtain the benchmarks on the $\mathrm{CpG}$ resolution level. For the region-wise evaluation, $\mathcal{D}_{\mathrm{TP}}$ is the set of predicted DMRs in which $>50 \%$ of the DMRs' CpGs are simulated to be differential, i.e., elements of $\mathcal{D}_{\text {sim. }}$. On the other hand, $\mathcal{D}_{\mathrm{TP}^{\prime}}$ is the set of simulated DMRs in which $>50 \%$ of the DMRs' CpGs are in $\mathcal{D}_{\mathrm{TP}}$. Given

$$
\begin{gathered}
\mathcal{D}_{\mathrm{TP}}=\left\{D \in \mathcal{D}_{\text {pred }} \mid \frac{\left|C_{\mathrm{TP}} \cap D\right|}{|D|}>0.5\right\}, \\
C_{\mathrm{TP}}^{\prime}={\underset{D \in \mathcal{D}_{\mathrm{TP}}}{\cup} D,}_{\mathcal{D}_{\mathrm{TP}^{\prime}}=\left\{D \in \mathcal{D}_{\text {sim }} \mid \frac{\left|C_{\mathrm{TP}}^{\prime} \cap D\right|}{|D|}>0.5\right\},},
\end{gathered}
$$

we calculate

$$
\begin{aligned}
& \operatorname{PPV}_{\text {DMR }}=\left|\mathcal{D}_{\mathrm{TP}}\right| /\left|\mathcal{D}_{\text {pred }}\right|, \\
& \operatorname{TPR}_{\mathrm{DMR}}=\left|\mathcal{D}_{\mathrm{TP}^{\prime}}\right| /\left|\mathcal{D}_{\text {sim }}\right|,
\end{aligned}
$$

to obtain the benchmarks for the region-wise comparison analogously. Note that the distinction between $\mathcal{D}_{\mathrm{TP}}$ and $\mathcal{D}_{\mathrm{TP}}$, is necessary due to a possible m-to-n rather than a 1-to-1 association between simulated and predicted DMRs.

\section{Accuracy of DMR boundaries}

Boundaries of predicted DMRs were compared to simulated DMRs. More precisely, the distance (in CpGs) between the boundaries of each simulated DMR and its closest correctly predicted (TP) DMR was calculated. To analyze and compare the accuracy regarding the boundary detection of the different segmentation tools, the empirical cumulative distribution function of the absolute values of these distances was used.

\section{Whole-genome bisulfite data of medulloblastoma tumors}

DNA methylation data of 12 human medulloblastoma tumors (subgroup 4) and eight human normal controls were taken from Hovestadt et al. (2014). The qualitative analysis of the WGBS data set with 12 versus eight samples $(22,524,970$ data points without missing values) was restricted to Chromosome 10 with a total of 1,111,583 methylation data points. All segmentation tools were 
run in their default settings; and for MOABS, the maximal distance between two consecutive DMCs to be considered in a DMR (maxDistConsDmcs option) was set to $300 \mathrm{nt}$ to be comparable to metilene and BSmooth. DMRs were required to contain at least 10 informative CpGs, i.e., CpGs with an associated methylation rate, and an absolute methylation difference (average MDS) larger than 0.1. As described above, outputs of MOABS and metilene were filtered with the critical $P$-value 0.05 . For the analysis of promoter downstream correlating regions (pdCRs), the full set of pdCRs published in Hovestadt et al. (2014) was downloaded and restricted to Chromosome 10. For each pdCR, the difference between the average methylation of the tumor samples and the average methylation of the control samples was calculated using BEDTools (Quinlan and Hall 2010).

\section{Evaluation of runtime and memory requirements}

The running time of all tools was measured using the Unix time command, while the maximal residual memory consumption (RAM) was tracked by the Unix ps command. The evaluation was done separately with 10 cores or one core of a cluster with the following specifications: Intel XEON E7540 CPU at $2.00 \mathrm{GHz} \times 24$, $520 \mathrm{~GB}$ RAM. To measure memory and runtime requirements for the WGBS data set, we used 10 cores of the same cluster machine. To evaluate metilene's performance in an environment with restricted computational resources, the WGBS data set was additionally processed on a desktop machine with the following specifications: Intel Core i5-4570 CPU at $3.20 \mathrm{GHz} \times 4,7.8 \mathrm{~GB}$ RAM.

\section{Software availability}

The software is published under the GNU GPL v2.0 license. The source code of metilene is available in the Supplemental Material and at http://www.bioinf.uni-leipzig.de/Software/metilene/. The implementation that was used for benchmarking (metilene version 0.2-4) as well as the scripts for simulating DMRs is also part of the Supplemental Material.

\section{Acknowledgments}

This publication is supported by the German Bundesministerium für Bildung und Forschung (BMBF; PTJ grant HNPCCSys 031 6065A and ICGC MMML-Seq 01KU1002J), the European Union in the framework of the BLUEPRINT (HEALTH-F5-2011-282510) project, and by LIFE: Leipzig Research Center for Civilization Diseases, University of Leipzig. LIFE is funded by means of the European Union, by the European Regional Development Fund (ERDF), the European Social Fund (ESF), and by means of the Free State of Saxony within the framework of the excellence initiative.

Author contributions: F.J., H.K., P.F.S., and S.H. conceived the project. F.J. and S.H. implemented metilene. H.K., F.J., C.O., and S.H.B. tested metilene on artificial and biological data. F.J., H.K., and S.H. wrote the manuscript with contributions from all authors.

\section{References}

Akalin A, Kormaksson M, Li S, Garrett-Bakelman FE, Figueroa ME, Melnick A, Mason CE. 2012. methylkit: a comprehensive R package for the analysis of genome-wide DNA methylation profiles. Genome Biol 13: R87.

Bell J, Loomis A, Butcher L, Gao F, Zhang B, Hyde C, Sun J, Wu H, Ward K, Harris J, et al. 2014. Differential methylation of the TRPA1 promoter in pain sensitivity. Nat Commun 5: 2978.

Cortese R, Lewin J, Bäckdahl L, Krispin M, Wasserkort R, Eckhardt F, Beck S. 2011. Genome-wide screen for differential DNA methylation associated with neural cell differentiation in mouse. PLoS One 6: e26002.
Day K, Waite L, Thalacker-Mercer A, West A, Bamman M, Brooks J, Myers R, Absher D. 2013. Differential DNA methylation with age displays both common and dynamic features across human tissues that are influenced by CpG landscape. Genome Biol 14: R102.

De Jager PL, Srivastava G, Lunnon K, Burgess J, Schalkwyk LC, Yu L, Eaton ML, Keenan BT, Ernst J, McCabe C, et al. 2014. Alzheimer's disease: early alterations in brain DNA methylation at ANK1, BIN1, RHBDF2 and other loci. Nat Neurosci 17: 1156-1163.

Ernst J, Kellis M. 2010. Discovery and characterization of chromatin states for systematic annotation of the human genome. Nature Biotechnol 28: $817-825$.

Ernst J, Kheradpour P, Mikkelsen TS, Shoresh N, Ward LD, Epstein CB, Zhang X, Wang L, Issner R, Coyne M, et al. 2011. Mapping and analysis of chromatin state dynamics in nine human cell types. Nature 473: 43-49.

Fasano G, Franceschini A. 1987. A multidimensional version of the Kolmogorov-Smirnov test. Mon Not R Astron Soc 225: 155-170.

Hansen KD, Langmead B, Irizarry RA. 2012. BSmooth: from whole genome bisulfite sequencing reads to differentially methylated regions. Genome Biol 13: R83.

Hebestreit K, Dugas M, Klein HU. 2013. Detection of significantly differentially methylated regions in targeted bisulfite sequencing data. Bioinformatics 29: 1647-1653.

Hovestadt V, Jones DT, Picelli S, Wang W, Kool M, Northcott PA, Sultan M, Stachurski K, Ryzhova M, Warnatz HJ, et al. 2014. Decoding the regulatory landscape of medulloblastoma using DNA methylation sequencing. Nature 510: 537-541.

Kretzmer H, Bernhart SH, Wang W, Haake A, Weniger MA, Bergmann AK, Betts MJ, Carrillo-de Santa-Pau E, Doose G, Gutwein J, et al. 2015. DNA methylome analysis in Burkitt and follicular lymphomas identifies differentially methylated regions linked to somatic mutation and transcriptional control. Nat Genet 47: 1316-1325.

Nilsson E, Jansson PA, Perfilyev A, Volkov P, Pedersen M, Svensson MK, Poulsen P, Ribel-Madsen R, Pedersen NL, Almgren P, et al. 2014. Altered DNA methylation and differential expression of genes influencing metabolism and inflammation in adipose tissue from subjects with type 2 diabetes. Diabetes 63: 2962-2976.

Olshen AB, Venkatraman E, Lucito R, Wigler M. 2004. Circular binary segmentation for the analysis of array-based DNA copy number data. Biostatistics 5: 557-572.

Park Y, Figueroa ME, Rozek LS, Sartor MA. 2014. MethylSig: a whole genome DNA methylation analysis pipeline. Bioinformatics 30: 2414-2422.

Quinlan AR, Hall IM. 2010. BedTools: a flexible suite of utilities for comparing genomic features. Bioinformatics 26: 841-842.

R Core Team. 2013. R: a language and environment for statistical computing. $\mathrm{R}$ Foundation for Statistical Computing, Vienna, Austria. http://www.Rproject.org/.

Raney BJ, Cline MS, Rosenbloom KR, Dreszer TR, Learned K, Barber GP, Meyer LR, Sloan CA, Malladi VS, Roskin KM, et al. 2011. ENCODE whole-genome data in the UCSC genome browser (2011 update). Nucleic Acids Res 39: D871-D875.

Sheaffer KL, Kim R, Aoki R, Elliott EN, Schug J, Burger L, Schübeler D, Kaestner KH. 2014. DNA methylation is required for the control of stem cell differentiation in the small intestine. Genes Dev 28: 652-664.

Siegmund DO. 1986. Boundary crossing probabilities and statistical applications. Ann Statist 14: 361-404.

Stockwell PA, Chatterjee A, Rodger EJ, Morison IM. 2014. DMAP: differential methylation analysis package for RRBS and WGBS data. Bioinformatics 30: 1814-1822.

Sun D, Xi Y, Rodriguez B, Park HJ, Tong P, Meong M, Goodell MA, Li W. 2014. MOABS: model based analysis of bisulfite sequencing data. Genome Biol 15: 38.

Teschendorff AE, Menon U, Gentry-Maharaj A, Ramus SJ, Weisenberger DJ, Shen H, Campan M, Noushmehr H, Bell CG, Maxwell AP, et al. 2010 Age-dependent DNA methylation of genes that are suppressed in stem cells is a hallmark of cancer. Genome Res 20: 440-446.

Teschendorff AE, Yang Z, Wong A, Pipinikas CP, Jiao Y, Jones A, Anjum S, Hardy R, Salvesen HB, Thirlwell C, et al. 2015. Correlation of smoking-associated DNA methylation changes in buccal cells with DNA methylation changes in epithelial cancer. JAMA Oncol 1: 476-485.

Zhang NR, Siegmund DO. 2012. Model selection for high-dimensional multi-sequence change-point problems. Stat Sin 22: 2507-1538.

Ziller MJ, Hansen KD, Meissner A, Aryee MJ. 2015. Coverage recommendations for methylation analysis by whole-genome bisulfite sequencing. Nat Methods 12: 230-232.

Received July 1, 2015; accepted in revised form November 25, 2015.

\section{Genome Research}

www.genome.org 


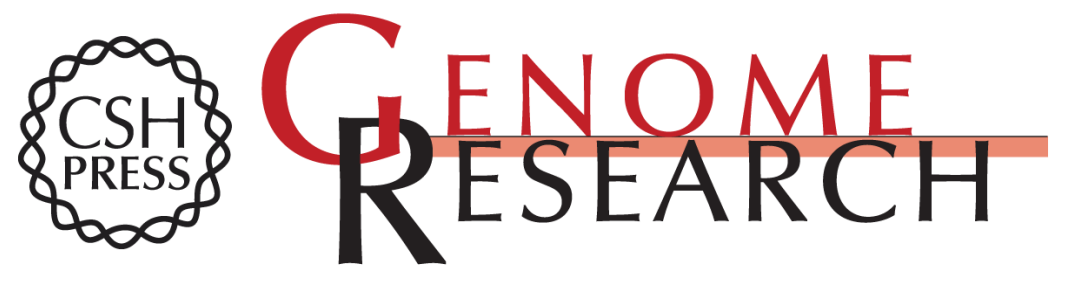

\section{metilene: fast and sensitive calling of differentially methylated regions from bisulfite sequencing data}

Frank Jühling, Helene Kretzmer, Stephan H. Bernhart, et al.

Genome Res. 2016 26: 256-262 originally published online December 2, 2015

Access the most recent version at doi:10.1101/gr.196394.115

Supplemental Material

References

Creative

Commons

License

Email Alerting

Service
http://genome.cshlp.org/content/suppl/2015/12/07/gr.196394.115.DC1

This article cites 25 articles, 3 of which can be accessed free at: http://genome.cshlp.org/content/26/2/256.full.html\#ref-list-1

This article is distributed exclusively by Cold Spring Harbor Laboratory Press for the first six months after the full-issue publication date (see

http://genome.cshlp.org/site/misc/terms.xhtml). After six months, it is available under a Creative Commons License (Attribution-NonCommercial 4.0 International), as described at http://creativecommons.org/licenses/by-nc/4.0/.

Receive free email alerts when new articles cite this article - sign up in the box at the top right corner of the article or click here.

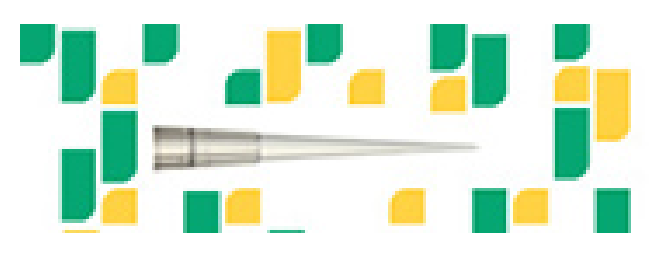

Focused on your science.

Jコగ

SCIENTIFIC

saos or seisnes

To subscribe to Genome Research go to:

https://genome.cshlp.org/subscriptions 